\title{
Pronominal Anaphor Resolution and the Underlying Cognitive Mechanism
}

\author{
Huijun Tang ${ }^{1,2 *}$, Wei Li ${ }^{1}$ \\ ${ }^{1}$ College of International Studies, Southwest University, Chongqing, China \\ ${ }^{2}$ College of Foreign Languages and Culture, Chengdu University, Chengdu, China \\ Email: ^helenaccnu@hotmail.com
}

How to cite this paper: Tang, H. J., \& Li, W. (2018). Pronominal Anaphor Resolution and the Underlying Cognitive Mechanism. Open Journal of Modern Linguistics, 8, 9-16. https://doi.org/10.4236/ojml.2018.82002

Received: February 6, 2018

Accepted: April 8, 2018

Published: April 11, 2018

Copyright (c) 2018 by authors and Scientific Research Publishing Inc. This work is licensed under the Creative Commons Attribution International License (CC BY 4.0).

http://creativecommons.org/licenses/by/4.0/

\section{c) (i) Open Access}

\begin{abstract}
When people tend to refer to a previous mentioned discourse entity, they can use various types of anaphoric expressions, such as pronouns, definite noun phrases, proper names or expressions that are introduced by a lexical modifier or a comparative adjective. Among the different types of anaphoric expressions, pronoun is most common and frequently used which contains only a little information about the referent, while definite expressions contain much richer semantic information. As pronouns contain very little information on their own, they are always ambiguous. The cognitive mechanism that underlies the comprehension of pronouns, especially the ones with ambiguities, catches the interests of many researchers. Various tasks have been used to investigate this issue and the results did not go for the same direction. A combination of multiple research methods is needed for a better and deeper understanding of anaphora.
\end{abstract}

\section{Keywords}

Anaphora, Resolution, Ambiguity, Mechanism

\section{Introduction}

In order to successfully comprehend a text, language users always have to determine whether expressions in different parts of the text refer to the same discourse entity. A common way to establish this kind of referential relations is to use anaphora. Anaphora refers to the linguistic relation that a linguistic element refers to another linguistic element in a text which usually occurs before it. The linguistic element which refers to another element (the anaphoric form) is called anaphor and the element that the anaphor refers to is called antecedent. The interpretation of the anaphoric expression is usually dependent on the interpreta- 
tion of its antecedents. For example, in sentence "When Michael finished eating, he put away the plate and cutlery", the pronoun "he" is an anaphor, referring back to the proper name "Michael", who serve as the antecedent of the anaphor. The interpretation of an anaphor is somewhat dependent on its antecedent. The process of determining the antecedent of an anaphor is referred as anaphora resolution. An interesting question is what affects people's choices of anaphoric expressions in language production and what affects peoples' resolution of anaphora in language comprehension? This has motivated much research in the field of functional linguistic, psycholinguistic, cognitive linguistic, computational linguistic, etc.

\section{Different Types of Anaphora}

\subsection{Pronominal Anaphora}

The most frequent type of anaphora is the pronominal anaphora, which is characterized by anaphoric pronouns. Pronouns form a special class of anaphors because of their empty semantic structure, i.e. they do not have an independent meaning from their antecedent.

1) Mary said that she would come home late tonight.

Sentence (1) is an example of pronominal anaphora in which the pronoun "she" need to be referred back to the previously mentioned linguistic entity "Mary" for its interpretation.

\subsection{Lexical Anaphora}

Lexical anaphora is sometimes called "definite noun phrase anaphora" (Mitkov, 1999). It is identified when the referring expressions are definite descriptions or proper nouns.

2) Ten university students were enrolled in the experiment. The participants are all native speakers of Mandarin Chinese.

In this example, "The participants" at the beginning of the second sentence refers back to "Ten university students" which is a definite noun phrase that represents a semantically close concept.

\subsection{Comparative Anaphora}

The comparative anaphora is when the anaphoric expressions are introduced by lexical modifiers (e.g. other, another) or comparative adjectives (e.g. better, greater). The "one anaphora" is a type of comparative anaphora and there are many researchers who focus on the "one anaphora" (Mitkov, 1999).

3) The girl planted a rose next to the yellow one.

Sentence (3) is an example of comparative anaphora in which the word "one" refers to the word "rose".

\subsection{Complement Anaphora}

There are cases in which the anaphoric expressions do not refer to their normal 
antecedents, but instead, the complement of the antecedent. This type of anaphora is called complement anaphora. Take sentence (4) as an example.

4) Most of the students have passed the language test. They need resit the test next semester.

In this sentence, the anaphor "they" does not refer to the anteceding "most of the students". Contrastively, the anaphor refers to the complement set of "most of the students", which is "the rest of the students who did not pass the language test".

\section{Factors Affecting Anaphora Resolution}

There are several factors that affect anaphora resolution, such as the distance between an anaphoric expression and its antecedent, gender and number agreement, grammatical role and superficial position of the antecedent, word frequency, structural parallelism, etc. Among the factors that underlie anaphora resolution, the accessibility of the antecedent and the gender coherence between the anaphor and the antecedent have been proved to be very affective. In addition, the depth of processing also plays an important role of the building up of people's linguistic representations in the course of reference processing.

\subsection{Accessibility}

There is a general assumption that the choice of anaphor depends on the referent's accessibility or how activated it is in the discourse representation. When the referent is highly accessible in the discourse, reduced anaphoric expressions such as pronouns are preferred, while when the referent is less accessible, more explicit anaphors such as definite descriptions and proper names are tend to be used (Ariel, 1991; Gundel et al., 1993). According to the Accessibility theory (Ariel, 2006), highly informative anaphors, normally known as low accessibility makers, are easier to process when the antecedent is of low accessibility and are harder to process when the antecedent is of high accessibility. There are many factors that could affect accessibility and hence the choice of anaphoric expressions, such as the position of the referent (how close is the referent located to the anaphor in discourse), the frequency of referent's appearance, the grammatical role of the referent. These factors affect not only the choice of anaphoric expressions in language production but also anaphora resolution in language comprehension.

\subsection{Gender Coherence}

Another important factor that affects anaphora resolution is the gender in formation of the referents in discourse. Computational linguists consider gender agreement has as an affective eliminating factor which can help to exclude certain NPs from the set of possible antecedent candidates (Seddik \& Farghaly, 2014). When the gender of the anaphor and the antecedent do not agree with each other, the processing difficulties occurs and if the gender in formation of 
the anaphor agree with more than one preceding discourse entities, there would be ambiguities. As pronouns contained only limited information, they could always be ambiguous. Pronoun ambiguity has been intensively studied by linguists (Garnham, 2001 for an overview) and results differ. Some argue that pronoun ambiguity resolution involves in a competition of different antecedents (e.g., Gernsbacher, 1989; MacDonald \& McWhinney, 1995), while some other believed that ambiguity resolution employs a mechanism of reanalysis, in which a single interpretation is initially adopted and a reanalysis procedure occurs only when the initial interpretation is proved to be wrong (e.g., Traxler et al., 1998; Van Gompel et al., 2005). For more explicit anaphoric expressions which would not normally cause ambiguities, the gender in formation issue are seldom investigated. However, there might be some interconnection between the resolution of different types of anaphora and a more general processing mechanism may interpret anaphora resolution. Hence, it motivates the investigation of gender information of proper name anaphors in the current study.

\subsection{Depth of Processing}

When processing language in reading or listening, people tend to build up linguistic representations not only among the component linguistic entities within a certain sentence, but also representation between sentences which connect the components and the utterances. Regarding the way of building up the linguistic representations, different ideas are held by researchers. According to the traditional theories of language processing, the linguistic representations that are built up by the language processors are precise and accurate. However, many contemporary researches have showed that these linguistic representations may not need to be precise in many circumstances. The idea of this account is that the linguistic representations built up in language processing are only need to be good enough for the task at hand and goes to a more precise stage of processing only when the task at hand requires the language processor to do so (Christianson et al., 2001; Stewart et al., 2007). Stewart and college (2007) have found in their experiment that in a shallow processing condition where the task did not require the participants to answer pronoun-related comprehension questions, sentences with ambiguous pronouns are processed at the same speed as the sentences with unambiguous pronouns. However, in a deep processing condition where the participants had to answer a pronoun-related comprehension question after every sentence, the processing time of the ambiguous pronoun sentence was significantly longer than the ones with unambiguous pronouns.

\section{Anaphoric Ambiguity Resolution}

When people refer to a previous mentioned discourse entity, they can use various types of anaphoric expressions, such as pronouns (e.g., he, him, his, this), definite noun phrase (e.g., the president, green tea), proper names (e.g., China, 
Mary) or expressions that are introduced by a lexical modifier or a comparative adjective (e.g., "one" in "another one"). Among the different types of anaphoric expressions, pronoun is most common and frequently used which contains only a little information about the referent, while definite expressions contain much richer semantic information.

\subsection{Ambiguity and Pronominal Anaphor}

Pronouns contain very limited information about their antecedent such as gender, number and animacy, and they are therefore frequently ambiguous. Many researchers have investigated the ambiguous pronoun resolution in language processing and results differed. There are two competing accounts in research on ambiguity. According to one account, a mechanism of competition is involved in ambiguity resolution which results in ambiguous words or sentences being harder to understand than unambiguous words or sentences. However, the other account assumes that only one analysis is initially activated in sentence processing and reanalysis occurs only when the initial analysis is found to be implausible. According to this reanalysis-based account, sentences processing are easy when there are ambiguities, compared to sentences without ambiguity. However, it is currently unclear which of the models best explains ambiguous pronoun resolution, because research has shown conflicting results.

\subsection{Empirical Studies of Anaphoric Ambiguity}

The resolution of anaphors involves different mechanisms according to the competition theory and the reanalysis theory. In MacDonald and MacWhinney's (1990) study, two cross modal experiments were conducted to investigate changes in activation levels for pronominal referents and nonreferents. They measured participants' response times to visual probe words when they listened to sentences. They found that in the ambiguous pronoun condition in which the pronoun refers to two antecedent name of the same gender, response times to both the referent probe word and nonreferent probe word were generally slower than in the no-pronoun control condition. However, a significant pronoun facilitation effect for the referent was found in the unambiguous reference condition, when comparing the pronoun condition to the no-pronoun baseline. No reaction time difference between the Pronoun condition and the No-pronoun condition was found in the unambiguous pronoun situation. These results suggest that ambiguous pronoun has an inhibiting effect on the response time, indicating that the ambiguity of the pronoun causes longer processing times. These are similar to the findings of Gernsbacher's (1989) study in which the researcher also found an inhibiting effect for the nonreferent. However, Gernsbacher also found the existence of facilitation effects, but they were weaker than the inhibiting effects. The MacDonald and MacWhinney's (1990) findings suggest that ambiguous pronouns are harder to comprehend than unambiguous pronouns. This is consistent with the theory of competition. 
Although most research has found evidence supporting the idea of competition, there were studies showed quite different results. Vonk (1985) carried out a study that deals with the immediacy of processing pronouns in relation to the purpose of reading. In their materials, the gender of the pronoun and of the antecedents was varied to create conditions with a gender cue and without a gender cue on the basis of which the pronoun could be unambiguously assigned. By using a verification task, Vonk found that the verification latencies showed an effect of both the congruency factor and the gender factor. She found that in the first pass through the sentence, the fixation time on the pronouns was longer when there was a gender cue and the pronoun was more often fixated when there was a gender cue. That is, when the pronoun was informative, it was fixated more often and for longer. This indicated that the informative pronouns cause difficulty in the processing which results in a longer fixation time. These findings fit a reanalysis model which predicts that only one analysis is initially activated and reanalysis occurs when the initial analysis is impossible, resulting in a longer reading time. According to this theory, the condition with a gender cue can involve a procedure of reanalysis and therefore a longer processing time than the condition with no gender cue. Rigalleau and Caplan (2000) found evidence for an immediate and automatic process that relates a pronoun to potential antecedents and the resolution of the pronoun involves a controlled process when the automatic process failed to converge on a single noun as the antecedent of the pronoun. This is also consistent with the theories of the reanalysis models.

\section{Discussion}

As noticed and explained in Vonk's (1985) study, different results on pronoun processing were obtained from different tasks. The results form a naming task (Vonk, 1984) differed a lot from the results from the verification task (Vonk, 1985) on the same research area. According to Vonk (1985), participants were very much addressed to the pronoun as they had to name aloud the antecedent of the pronoun, but in the verification task the participants focused much less on the pronoun. However, neither the naming task nor the verification task could be considered as normal language processing because they did not reflect people's normal reading behaviours. Some other studies may have similar limitations. In MacDonald and MacWhinney's (1995) study, participants were tested when listening to spoken words or sentences from a speaker and they had to make their decision about the target words on the basis of the information that they heard. This is very different from what people normally do during natural language processing. Hence the responses that were recorded and the data that was calculated cannot convincingly provide evidence for their priori assumptions and hypotheses. Since these tasks were not like normal language processing, participants may well have adopted certain kinds of strategies when doing the tasks. This may result in the activation of both the interpretations of the am- 
biguous words, and therefore competition will occur. In this case, the results they obtained may have been largely affected by the inappropriate experimental method. Any data collected from such a task and any analysis based on these data will possibly cause inaccurate results that cannot be generalised to natural reading.

\section{Conclusion}

Anaphora is a common way that people tend to use when producing natural language utterances and the comprehension of anaphors catches the interests of many researchers. The linguistic representations that people built in the course of anaphor processing are affected by many factors and many previous studies have investigated anaphora resolution widely and intensively. However, previous studies have shown very different results and it is hard to conclude the exact mechanisms that underlie the anaphor processing. In addition, different research methods that were employed could lead to very different results. Future research may combine the traditional research methods and some real-time research technique such as eye-tracking and ERPs for a more comprehensive understanding of anaphora. The time course and the exact processing pattern of the anaphoric expression will provide us with a clearer map of anaphora resolution and the cognitive mechanism.

\section{Acknowledgements}

This research is funded by the Sichuan Provincial Social Science Planning Project (SC15WY001) and Chengdu University Youth Research Fund (2080515013).

\section{References}

Ariel, M. (1991). The Function of Accessibility in a Theory of Grammar. Journal of Pragmatics, 16, 443-463. https://doi.org/10.1016/0378-2166(91)90136-L

Ariel, M. (2006). Accessibility Theory. In K. Brown (Ed.), Encyclopedia of Language \& Linguistics (pp. 15-18). Elsevier Ltd. https://doi.org/10.1016/B0-08-044854-2/04291-7

Christianson, K., et al. (2001). Thematic Roles Assigned along the Garden Path Linger. Cognitive Psychology, 42, 368-407. https://doi.org/10.1006/cogp.2001.0752

Garnham, A. (2001). Mental Models and the Interpretation of Anaphora. Hove: Psychology Press.

Gernsbacher, M. A. (1989). Mechanisms That Improve Referential Access. Cognition, 32, 99-156. https://doi.org/10.1016/0010-0277(89)90001-2

Gundel, J. K., Hedberg, N., \& Zacharski, R. (1993). Cognitive Status and the Form of Referring Expressions in Discourse. Language, 69, 274-307. https://doi.org/10.2307/416535

MacDonald, M. C., \& MacWhinney, B. (1990). Measuring Inhibition and Facilitation from Pronouns. Journal of Memory and Language, 29, 469-492. https://doi.org/10.1016/0749-596X(90)90067-A

McDonald, J. L., \& MacWhinney, B. (1995). The Time Course of Anaphor Resolution: Effects of Implicit Causality and Gender. Journal of Memory and Language, 34, 543-566. https://doi.org/10.1006/jmla.1995.1025 
Mitkov, R. (1999). Multilingual Anaphora Resolution. Machine Translation, 14, 281-299. https://doi.org/10.1023/A:1011184828072

Rigalleau, F., \& Caplan, D. (2000). Effects of Gender Marking in Pronominal Coindexation. Quarterly Journal of Experimental Psychology, 53A, 23-52. https://doi.org/10.1080/713755884

Seddik, K. M., \& Farghaly, A. (2014). Anaphora Resolution. In: I. Zitouni (Ed.), Natural Language Processing of Semitic Languages: Theory and Applications of Natural Language Processing (pp. 247-277). Berlin: Springer. https://doi.org/10.1007/978-3-642-45358-8_8

Stewart, A. J., Holler, J., \& Kidd, E. (2007). Shallow Processing of Ambiguous Pronouns: Evidence for Delay. Quarterly Journal of Experimental Psychology, 60, 1680-1696. https://doi.org/10.1080/17470210601160807

Traxler, M. J., Pickering, M. J., \& Clifton, C. (1998). Adjunct Attachment Is Not a Form of Lexical Ambiguity Resolution. Journal of Memory and Language, 39, 558-592. https://doi.org/10.1006/jmla.1998.2600

Van Gompel, R. P. G., Pickering, M. J., Pearson, J., \& Liversedge, S. P. (2005). Evidence against Competition during Syntactic Ambiguity Resolution. Journal of Memory and Language, 52, 284-307. https://doi.org/10.1016/j.jml.2004.11.003

Vonk, W. (1984). Eye Movements during the Comprehension of Pronouns. In A. G. Gale, \& F. Johnson (Eds.), Theoretical and Applied Aspects of Eye Movement Research (pp. 203-212). Amsterdam: Elsevier. https://doi.org/10.1016/S0166-4115(08)61835-X

Vonk, W. (1985). On the Purpose of Reading and the Immediacy of Processing Pronouns. In R. Groner, G. W. McConkie, \& C. Menz (Eds.), Eye Movements and Human Information Processing (pp. 207-215). Amsterdam: Elsevier. 